\title{
Effects of TiN Buffer Layer Thickness on GaN Growth
}

\section{AUTHOR(S):}

Ito, Kazuhiro; Uchida, Yu; Lee, Sangjin; Tsukimoto, Susumu; Ikemoto, Yuhei; Hirata, Koji; Murakami, Masanori

\section{CITATION:}

Ito, Kazuhiro ...[et al]. Effects of TiN Buffer Layer Thickness on GaN Growth. Journal of Electronic Materials 2009, 38(4): 511-517

\section{ISSUE DATE:}

2009-04

URL:

http://hdl.handle.net/2433/126667

\section{RIGHT:}

The original publication is available at www.springerlink.com; この論文 は出版社版でありません。引用の際には出版社版をご確認ご利用くだ さい。; This is not the published version. Please cite only the published version. 


\section{Effects of TiN Buffer Layer Thickness on GaN Growth}

Kazuhiro Ito, ${ }^{1}$ Yu Uchida, ${ }^{1}$ Sangjin Lee, ${ }^{1}$ Susumu Tsukimoto, ${ }^{1}$ Yuhei Ikemoto, ${ }^{2}$ Koji Hirata, $^{2}$ and Masanori Murakami ${ }^{1,3}$

1. Department of Materials Science and Engineering, Kyoto University, Kyoto 606-8501, Japan

2. Optoelectronics Division, Toyoda Gosei Co., Ltd. Inazawa, Aichi 490-1312, Japan

3. Present Address: The Ritsumeikan Trust, Nakagyo-ku, Kyoto 604-8520, Japan

\section{Abstract}

Smooth GaN layers were successfully grown on metallic TiN buffer layers by metal organic chemical vapor deposition (MOCVD). One important factor in controlling GaN layer smoothness was the TiN layer thickness. We investigated systematically the effects of this thickness, and found an optimal thickness of $5 \mathrm{~nm}$, at which the smallest average grain size $(20 \mathrm{~nm})$ and smoothest surface were obtained. The TiN layers increased surface coverage with GaN hexagons at an early stage of GaN growth, indicating that enhancing the GaN nucleation is essential for the smooth GaN layer growth, and small grain size and smooth surface are needed to enhance the GaN nucleation. Further reduction in TiN layer thickness to $2 \mathrm{~nm}$ decreased the surface coverage with GaN hexagons, and a high density of grooves and holes were observed in the surface of the 2 $\mu \mathrm{m}$-thick GaN layers. Defect-structures in the GaN layers grown on the TiN layers were remarkably changed on reduction of TiN layer thickness from $5 \mathrm{~nm}$ to $2 \mathrm{~nm}$. GaN growth was found to be sensitive to the TiN layer thickness between $2 \mathrm{~nm}$ and $5 \mathrm{~nm}$.

Key words: Epitaxial growth of GaN, TiN buffer layers, TiN layer thickness, dislocations 


\section{Introduction}

GaN-based semiconductors with wide bandgaps have been used as key elements in blue light-emitting diodes and laser diodes. As bulk GaN is not commercially available, non-conductive sapphires have been extensively used as substrates for GaN layers, grown by a metal organic chemical vapor deposition (MOCVD) technique. However, because there is a large lattice mismatch of about $15 \%$ between $\mathrm{GaN}$ and the sapphire substrates, a buffer layer to relax the lattice mismatch is needed for lateral growth of GaN on the sapphire substrates [1]. The best buffer layer in use was an AlN layer deposited at a relatively low temperature, and the use of this layer made it possible for epitaxial growth of the GaN layers with a mirror-like surface [2]. A disadvantage of using this AlN layer and sapphire substrate is that it is non-conductive. The use of a conductive buffer layer and substrate is desirable to increase the light emitting efficiency of the diodes [3]. As the first step in the development of a GaN growth technique using a conductive buffer layer and substrate, we attempted to develop a conductive buffer layer for epitaxial GaN layer growth.

We recently reported that epitaxial, continuous, flat GaN layers were successfully grown on metallic TiN buffer layers [4,5]. The purpose of our previous experiments was to study the effects of the TiN buffer layer on the quality of GaN layer growth, and three important conditions were found: (1) the TiN layer should have a-/b-axis crystal orientation, (2) the TiN layer should be nitrogen enriched, and (3) TiN layers of minimal thickness should be deposited at low substrate temperatures. The first two conditions were intensively studied in our previous experiments [4,5]. However, at that time we did not determine the optimal thickness of the buffer layer for growing the smoothest GaN layer. 
In the present study, we investigated the effects of layer thickness of the nitrogen-enriched TiN buffer layers on the smoothness of GaN growth. TiN layers with thicknesses in the range of $2 \mathrm{~nm}$ to $100 \mathrm{~nm}$ were deposited on sapphire substrates. The GaN growth mechanism on these TiN layers was studied by analyzing the microstructures observed using a scanning electron microscope (SEM), an atom force microscope (AFM), and a transmission electron microscope (TEM).

\section{Experimental Procedures}

TiN buffer layers with thicknesses in the range of $2 \mathrm{~nm}$ to $100 \mathrm{~nm}$ were deposited on the $(11 \overline{2} 0)$ sapphire plane at room temperature (RT) and $600^{\circ} \mathrm{C}$ by sputtering Ti targets with $6 \mathrm{~N}$ purity in a radio frequency magnetron sputtering system. The base pressure of the vacuum chamber was about $1 \times 10^{-7}$ Torr. The TiN layers were prepared using a flow ratio of $\mathrm{N}_{2}$ and Ar gases of 2 to 8 , and the vacuum chamber background pressure was maintained at about $3.5 \times 10^{-3}$ Torr during TiN deposition. The TiN layers deposited on the sapphire substrates were exposed to $\mathrm{NH}_{3}+\mathrm{H}_{2}$ mixed gas atmosphere at about $1000^{\circ} \mathrm{C}$ to enrich the nitrogen concentration of the TiN layers. GaN layers of about $0.2 \mu \mathrm{m}, 2 \mu \mathrm{m}$, or $5 \mu \mathrm{m}$ thicknesses were deposited on the nitrogen-enriched TiN layers using a MOCVD technique. The surfaces of the nitrogen-enriched TiN layers and as-deposited GaN layers were observed using SEM and AFM. The layer microstructures were analyzed using an X-ray diffraction (XRD) method and observed using TEM.

\section{Results and Discussion}

As-deposited TiN buffer layers were exposed to $\mathrm{NH}_{3}+\mathrm{H}_{2}$ mixed gas atmosphere to increase the nitrogen concentration of the layers. The nitrogen enrichment of the TiN 
buffer layers was found to bolster smooth GaN layer growth. The TiN grains were observed to grow during the nitrogen enrichment. Reducing the thickness of these nitrogen-enriched TiN buffer layers (hereafter called “TiN layers”) was also found to bolster smooth GaN layer growth. In the present study, we investigated effect of reducing the thickness of the TiN layers on the GaN layer growth, and its role in producing smooth GaN layers.

\section{Grain size and surface roughness at various TiN buffer layer thicknesses}

Figure 1 shows AFM surface images of TiN layers with thicknesses of $2 \mathrm{~nm}, 5 \mathrm{~nm}$, $20 \mathrm{~nm}$, and $100 \mathrm{~nm}$, which were deposited at RT. The average grain size of the TiN layers generally decreased with reduction in layer thickness. The grain size of the 5 nm-thick TiN layers was about $20 \mathrm{~nm}$, the smallest grains among the TiN layers tested. However, the grain size of the 2 nm-thick TiN layers was larger than that of the 5 nm-thick TiN layers.

The average grain size and surface roughness of the TiN layers deposited at RT and $600^{\circ} \mathrm{C}$ with various layer thicknesses are shown in Fig. 2. Both the average grain size and surface roughness of the TiN layers were decreased with reduction in layer thickness. Significant decrease in the average grain size and surface roughness was observed in the $5 \mathrm{~nm}$-thick TiN layers deposited at RT. Further reducing the thickness of the TiN layers did not further decrease the average grain size or surface roughness. Thus, the thickness of $5 \mathrm{~nm}$ was found to provide the smallest grain size and surface roughness among these thicknesses. Furthermore, smaller grain sizes were found to provide smoother surfaces. In addition, the average grain sizes of the TiN layers deposited at RT were smaller than those deposited at $600^{\circ} \mathrm{C}$. Thus, reducing substrate temperature was also found to decrease the average grain size. 


\section{Effect of reducing the TiN layer thickness on GaN growth}

To investigate the effect of reducing the thickness of the TiN layers on GaN growth, GaN layers were deposited on the $2 \mathrm{~nm}$-, $5 \mathrm{~nm}$-, and $10 \mathrm{~nm}$-thick TiN layers deposited at RT. SEM surface images of the $0.2 \mu \mathrm{m}$ and $2 \mu \mathrm{m}$-thick GaN layers are shown in Fig. 3. In the $0.2 \mu$ m-thick GaN layers (an early stage of GaN growth), GaN island-growth was observed in all the samples. Most of the $5 \mathrm{~nm}$-thick TiN layer was covered with GaN hexagons (Fig. 3b). GaN hexagon coverage of the TiN layers of other thicknesses was less complete (Figs. 3a and c). The surface coverage with GaN hexagons on the 10 nm-thick TiN layers (Fig. 3c) was similar to that on the 20 nm-thick TiN layers, and was larger than those on the TiN layers with thicknesses of more than $20 \mathrm{~nm}$. This indicates that the surface coverage with GaN hexagons was largest for the TiN layers of $5 \mathrm{~nm}$ thickness, and the TiN layer had the smallest grain size and smoothest surface. In addition, the size of GaN hexagons decreased simply with decreasing thickness of the TiN layers. The GaN hexagons grown on the 2 nm-thick TiN layer are remarkably small compared with those on the TiN layers with other thicknesses. This suggests that GaN growth tendency was critically changed when the thickness of the TiN layers was reduced from $5 \mathrm{~nm}$ to $2 \mathrm{~nm}$.

GaN layer growth was observed in all the samples at various $\mathrm{GaN}$ layer thicknesses. Figures 3d-f show SEM surface images of the $2 \mu \mathrm{m}$-thick GaN layers. Continuous and smooth surfaces were observed on the GaN layers grown on the $5 \mathrm{~nm}$ - and $10 \mathrm{~nm}$-thick TiN layers (Figs. 3e and f). In the GaN layers grown on the 10 nm-thick TiN layer, facets of the GaN hexagons were observed, and this was similar in the GaN layers grown on the 20 nm-thick TiN layers. These facets did not appear in the GaN layers grown on the 5 nm-thick TiN layers. In contrast, many grooves and holes were observed 
in the GaN layers grown on the 2 nm-thick TiN layer (Fig. 3d). These indicate that large surface coverage with GaN hexagons on the TiN layers in the early stage of GaN growth was essential for the smooth GaN layer growth, and that small grain size and smooth surface are needed for the large surface coverage with GaN hexagons.

The average surface roughness, $R$ a, observed in the $1 \mu \mathrm{m} \times 1 \mu \mathrm{m}$ area of the 2 $\mu$ m-thick GaN layers grown on the TiN layers at various layer thicknesses deposited at RT is shown in Fig. 4a. The Ra value decreased with decreasing TiN layer thickness, and reached the minimum value of about $30 \mathrm{~nm}$ in the GaN layers grown on the 5 nm-thick TiN layers. Further decrease of the TiN layer thickness increased the Ra value. The smoothest surface was obtained in the GaN layers grown on the 5 nm-thick TiN layers, which had the smallest grain size and smoothest surface. Figure $4 \mathrm{~b}$ shows surface roughness of the $2 \mu$ m-thick GaN layers when a $20 \mu \mathrm{m}$ line-scan was carried out. The spread of the roughness decreased with decreasing thickness of the TiN layers, and was the smallest in the $5 \mathrm{~nm}$-thick TiN layers. In contrast, many local depressions, corresponding to the grooves and holes, were observed in the GaN layers grown on the 2 nm-thick TiN layers. The dependence of thickness of the TiN layers on the surface roughness of the GaN layers is in good agreement with the SEM observations (Fig. 3). The best TiN layer thickness was found to be $5 \mathrm{~nm}$ for the smooth GaN growth. The GaN growth tendency was also found to be critically changed when the thickness of the TiN layers was reduced from $5 \mathrm{~nm}$ to $2 \mathrm{~nm}$.

\section{Characterization of the GaN layers grown on the TiN layers}

The lattice mismatch between GaN and TiN is about 6.2\%, and is larger than that between GaN and AlN (2.5\%). Although the lattice mismatch of 2.5\% is small, a high density of threading dislocations (about $10^{8}-10^{10} \mathrm{~cm}^{-2}$ ) was observed in the GaN layers 
grown on AlN. Thus, characterization of the GaN layers grown on TiN was carried out using XRD and TEM.

The $5 \mu$ m-thick GaN layers grown on the 20 nm-thick TiN layers provided half-value widths of about 2500”-3000” and 700”-1000” for the XRD ( $\omega$-scan) profiles of the (0002) and $\{10 \overline{1} 0\}$ planes, respectively, as shown in Fig. 5a and 5b. The large half-value width of the (0002) profile was characteristic of the GaN layer grown on the TiN layers. The (0002) $\omega$-scan profile was recognized to be trapezoidal in shape. The (0002) $\omega$-scan was carried out in the way that $2 \theta$ was fixed in the distance of the (0002) plane, and $\theta$ was swung. Thus, the trapezoidal shape suggested that there are some [0001]-oriented grains where the [0001] direction is deviated from the direction perpendicular to the substrates, as shown schematically in Fig. 5a. In contrast, the $\{10 \overline{1} 0\} \omega$-scan profile suggested no such the deviation in terms of $<10 \overline{1} 0>$ directions. Thus, the "tilt" of the [0001]-oriented GaN grains is more remarkable than their "twist", and that large lattice mismatch between GaN and TiN is relaxed partly by the "tilt" of the GaN grains. The trapezoid shape of the (0002) $\omega$-scan profiles was often observed when the thickness of the TiN layers was greater than $10 \mathrm{~nm}$. Typical (0002) $\omega$-scan profiles are shown in Fig. 6a. The values of the half-value width of those profiles were more than $1^{\circ}\left(3600^{\prime \prime}\right)$ in the GaN layers grown on the TiN layers with thicknesses more than $10 \mathrm{~nm}$, as shown in Fig. 6b. However, the values were significantly lower for the TiN layer thickness less than $5 \mathrm{~nm}$ (Fig. 6b). Thus, the thickness of the TiN layers plays an important role in the relaxation mechanisms.

Figures $7 \mathrm{a}$ and $\mathrm{b}$ show cross-sectional TEM images of the $2 \mu \mathrm{m}$-thick GaN layer grown on the 5 nm-thick TiN layer taken with $\boldsymbol{g}=0002$ and $11 \overline{2} 0$, respectively. In $\boldsymbol{g}=0002$ (Fig. 7a), the vertical lines are likely due to domains with opposite lattice polarity. Although some of the threading dislocations in the GaN layers grown on the 
AlN layers were in contrast for $\boldsymbol{g}=0002$, dislocation contrast was hardly observed in Fig. 7a. In $\boldsymbol{g}=11 \overline{2} 0$ (Fig. 7b), horizontal dislocations were observed, and their density was estimated to be about $10^{8}-10^{9} \mathrm{~cm}^{-2}$. The horizontal dislocations were out of contrast for $\boldsymbol{g}=0002$, and thus their Burger's vector $(\boldsymbol{b})$ is perpendicular to [0001]. Thus, the large lattice mismatch between $\mathrm{GaN}$ and TiN would be relaxed partly by dislocations in the GaN layers. Note that the dislocation structure observed in the GaN layers grown on the TiN layers with the thickness of $5 \mathrm{~nm}$ or thicker is very different from that grown on the AlN buffer layers. The GaN layers grown on AlN were reported to have many threading dislocations, and they are edge or mixed [6]. However, density of threading dislocations is significantly reduced when TiN was used as a buffer layer. The GaN layers grown on TiN with the thickness of $5 \mathrm{~nm}$ or thicker have many horizontal dislocations, and they are screw, edge and mixed.

The defect-structure of the GaN layers was similar when the thickness of the TiN layers was $5 \mathrm{~nm}$ or thicker. The density of the horizontal dislocations and domains with opposite lattice polarity did not significantly depend on the thickness of the TiN layers if it is $5 \mathrm{~nm}$ or thicker. In contrast, the defect-structure was remarkably changed when thickness of the TiN layer was reduced from $5 \mathrm{~nm}$ to $2 \mathrm{~nm}$. Figures 7c and d show cross-sectional TEM images of the $2 \mu$ m-thick GaN layer grown on the 2 nm-thick TiN layer taken with $\boldsymbol{g}=0002$ and $11 \overline{2} 0$, respectively. The density of the threading dislocations is extremely high, as shown in Fig. 7c. They were in contrast for $\boldsymbol{g}=0002$ (Fig. 7c), but out of contrast for $\boldsymbol{g}=11 \overline{2} 0$ (Fig. 7d). Thus, they are screw dislocations. Since the value of the half-value width of the (0002) $\omega$-scan profiles was small, the large lattice mismatch would be relaxed mainly by threading dislocations in GaN layers. In addition to the many threading dislocations, a small number of horizontal and threading dislocations were observed in Fig. $7 d$. They were in contrast for $\boldsymbol{g}=11 \overline{2} 0$, but 
out of contrast for $\boldsymbol{g}=0002$, and thus their $\boldsymbol{b}$ is perpendicular to [0001]. Thus, horizontal dislocations are screw, edge and mixed, and the threading dislocations are edge. Such difference in defect-structures between the GaN layers grown on the $2 \mathrm{~nm}$ - and 5 nm-thick TiN layers is consistent with the above SEM and AFM observations (Figs. 4 and 5). Note that the dislocation structure observed in the GaN layers grown on the 2 nm-thick TiN layers is different from that grown on the AlN buffer layers. Both the GaN layers grown on the 2 nm-thick TiN layers and the AlN layers [6] have many threading dislocations. However, the density in the GaN/TiN system is higher than that in the GaN/AlN system. The threading dislocations are screw in the GaN/TiN system, but they are edge and mixed in the GaN/AlN system.

\section{Roles of the TiN layer thickness in obtaining smooth GaN growth}

Reducing the thickness of the TiN layers was essential for growth of smooth GaN layers. It enhanced surface coverage with GaN hexagons on the TiN layers, indicating enhancement of GaN nucleation. The reduction in thickness of the TiN layers to $5 \mathrm{~nm}$ resulted in smaller TiN grain size. As a grain boundary can become a nucleation site of GaN hexagons, the largest GaN nucleation can be explained partly by presence of the largest number of nucleation sites in the TiN layer with its thickness of $5 \mathrm{~nm}$.

The GaN nucleation increases in frequency also by decreasing the wetting angle of a GaN nucleus on the TiN layer. Figure 8 shows schematic illustrations of GaN nucleation dependence on the TiN layer thickness. The energy balance in one GaN nucleus formed on the TiN layer is shown in Fig. 8c:

$$
\gamma_{2}=\gamma_{1}+\gamma_{0} \cos \theta
$$

where $\gamma_{0}, \gamma_{1}, \gamma_{2}$, and $\theta$ are the surface energy of the GaN nuclus, the GaN/TiN interface energy, surface energy of the TiN layer, and the wetting angle, respectively. The 
frequency of the GaN nucleation is given by:

$$
n=n_{\mathrm{s}} \exp \left(-\Delta G^{*} / \mathrm{RT}\right)
$$

where $n_{\mathrm{s}}$ is the number of nucleation sites, and $\Delta G^{*}$ is the critical Gibbs free energy difference, giving:

$$
\begin{aligned}
& \Delta G^{*}=\left(16 \pi \mathrm{f}(\theta) \gamma_{0}{ }^{3}\right) /\left(3 \Delta G_{\mathrm{v}}{ }^{2}\right) \\
& \mathrm{f}(\theta)=\left(2-3 \cos \theta+\cos ^{3} \theta\right) / 4
\end{aligned}
$$

where $\Delta G_{\mathrm{v}}$ is the Gibbs free energy difference forming a GaN nucleus per unit volume. Thus, decreasing the wetting angle decreases the values of both $\mathrm{f}(\theta)$ and $\Delta G^{*}$, resulting in an increase the GaN nucleation frequency.

The lattice mismatch between TiN and sapphire is larger than that between $\mathrm{GaN}$ and TiN. Thus, reducing the thickness of the TiN layers to $5 \mathrm{~nm}$ may increase the strain energy, making the values of $\gamma_{1}$ and $\gamma_{2}$ become smaller and larger, respectively. This results in a smaller value of $\theta$ (Fig. $8 \mathrm{~d}->8 \mathrm{~b}$ ). Thus, the largest GaN nucleation was explained partly by the largest GaN nucleation frequency in the TiN layer thickness of 5 $\mathrm{nm}$ in terms of the wetting angle. In contrast, further reduction the thickness of the TiN layers to $2 \mathrm{~nm}$ changed the defect-structure of the GaN layers (Fig. 7). This may be caused by a corresponding change in the strain energy in the TiN layers. Based on the SEM surface images of the $0.2 \mu$ m-thick GaN layers (Fig. 3a), values of $\gamma_{1}$ and $\gamma_{2}$ were expected to become larger and smaller, respectively, and this results in a larger value of $\theta$ (Fig. 8a). This suggests that the strain energy in the 2 nm-thick TiN layers may become smaller than those in the TiN layers with thicknesses of more than $5 \mathrm{~nm}$.

\section{Conclusions}

The average grain size and surface roughness of the 5 nm-thick TiN layer was smallest among the TiN layers with thicknesses in the range of $2 \mathrm{~nm}$ to $100 \mathrm{~nm}$. Most of 
the surface of the $5 \mathrm{~nm}$-thick TiN layer was covered with GaN hexagons in an early stage of GaN growth $(0.2 \mu$ m-thick GaN layers). The $2 \mu$ m-thick GaN layer grown on the $5 \mathrm{~nm}$-thick TiN layer exhibited the smoothest GaN surface, with Ra of about $30 \mathrm{~nm}$. Thus, the TiN layer thickness of $5 \mathrm{~nm}$ is believed to be the best for smooth GaN growth on TiN layers. This indicates that enhancing the $\mathrm{GaN}$ nucleation was essential for the smooth GaN layer growth, and that small grain size and smooth surface are needed for the enhancing the GaN nucleation. Further reduction of the TiN layer thickness down to $2 \mathrm{~nm}$ decreased the surface coverage with GaN hexagons, and a high density of grooves and holes were observed in the surface of the $2 \mu$ m-thick GaN layers.

Horizontal dislocations were observed in the GaN layers grown on the 5 nm-thick TiN layers. In addition, the domains with opposite lattice polarity were observed. These observations were characteristic of the GaN layers grown on the TiN layers with thicknesses of more than $5 \mathrm{~nm}$. In contrast, defect-structures in the GaN layers grown on the TiN layers were remarkably changed when the thickness of the TiN layer was reduced from $5 \mathrm{~nm}$ to $2 \mathrm{~nm}$. Threading dislocations were mainly observed. The GaN growth mechanism was found to be sensitive to the TiN layer thickness between $2 \mathrm{~nm}$ and $5 \mathrm{~nm}$.

\section{Acknowledgements}

This work was supported by Grants-in-Aid for Scientific Research (No. 17760568 and 20656112) and Grants for Regional Science and Technology Promotion from The Ministry of Education, Culture, Sports, Science and Technology.

\section{References}

1) K.N. Tu, J.W. Mayer, L.C. Feldman, Electronic Thin Film Science, Macmillan, New 
York, 1992.

2) H. Amano, N. Sawaki and I. Akasaki, Appl. Phys. Lett., 48, 353 (1986).

3) N. Koide, H. Kato, M. Sassa, S. Yamasaki, K. Manabe, M. Hashimoto, H. Amano, K. Hiramatsu and I. Akasaki, J. Cryst. Growth, 115, 639 (1991).

4) T. Watanabe, K. Ito, S. Tsukimoto, Y. Ushida, M. Moriyama, N. Shibata and M. Murakami, Mater. Trans. 46, 1975 (2005).

5) Y. Uchida, K. Ito, S. Tsukimoto, Y. Ikemoto, K. Hirata, N. Shibata and M. Murakami, J. Electronic Mater. 35, 1806 (2006).

6) X.H. Wu, L.M. Brown, D. Kapolnek, S. Keller, S.P. DenBaars, and J.S. Speck, J. Appl. Phys. 80, 3228 (1996) 
Figure captions

Fig. 1 AFM surface images of nitrogen-enriched TiN layers with thicknesses of (a) $2 \mathrm{~nm}$, (b) $5 \mathrm{~nm}$, (c) $20 \mathrm{~nm}$, and (d) $100 \mathrm{~nm}$.

Fig. 2 Layer thickness dependence of average grain size and average surface roughness of nitrogen-enriched TiN layers deposited at RT and $600^{\circ} \mathrm{C}$.

Fig. 3 SEM surface images of (a)-(c) $0.2 \mu \mathrm{m}$ - and (d)-(f) $2 \mu \mathrm{m}$-thick GaN layers grown on TiN layers with layer thicknesses of $2 \mathrm{~nm}, 5 \mathrm{~nm}$, and $10 \mathrm{~nm}$.

Fig. 4 (a) TiN layer thickness dependence of surface roughness of $2 \mu$ m-thick GaN layers, and (b) GaN surface roughness-scan length curves of the $2 \mu \mathrm{m}$-thick GaN layers grown on 2 nm-, 5 nm, 10 nm-, and 20nm-thick TiN layers.

Fig. 5 Typical XRD ( $\omega$-scan) profiles of the (0002) and $\{10 \overline{1} 0\}$ planes of $5 \mu$ m-thick GaN layers grown on 20 nm-thick TiN layers.

Fig. 6 (a) XED ( $\omega$-scan) profiles of the (0002) plane of $2 \mu$ m-thick GaN layers grown on TiN layers of various thicknesses. (b) Half-value width of the (0002) GaN diffraction peaks plotted as a function of the TiN layer thickness. The TiN layers were deposited at $\mathrm{RT}$ and $600^{\circ} \mathrm{C}$.

Fig. 7 TEM cross-sectional images of the $2 \mu \mathrm{m}$-thick GaN layers grown on (a) and (b) 5 nm-, and (c) and (d) 2 nm-thick TiN layers. The TEM images were taken with (a) and 
(c) $\boldsymbol{g}=0002$, and (b) and (d) $\boldsymbol{g}=11 \overline{2} 0$.

Fig. 8 Schematic illustrations of GaN nucleation dependence on the TiN layer thickness. 

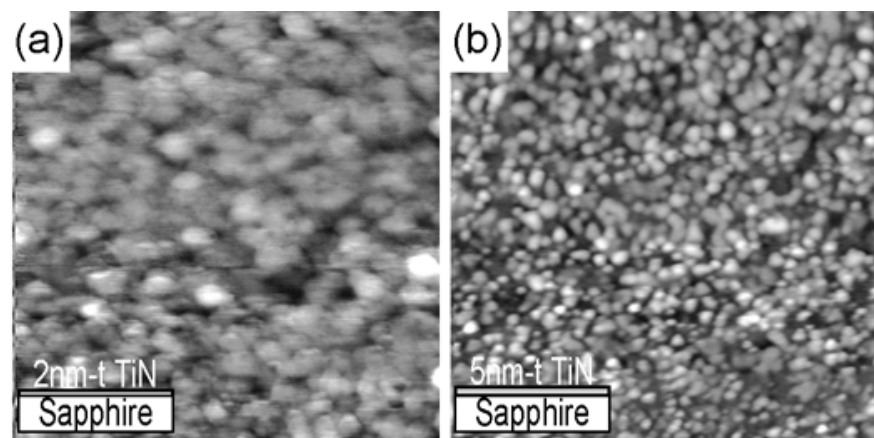

(c)

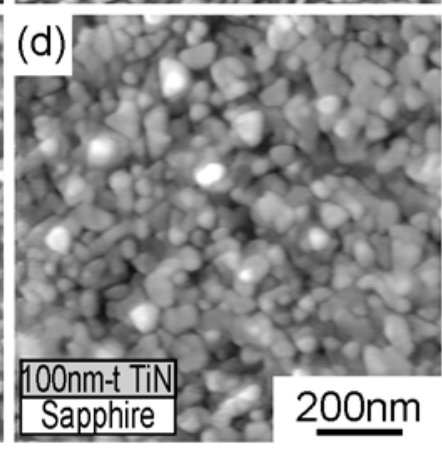

Fig. 1

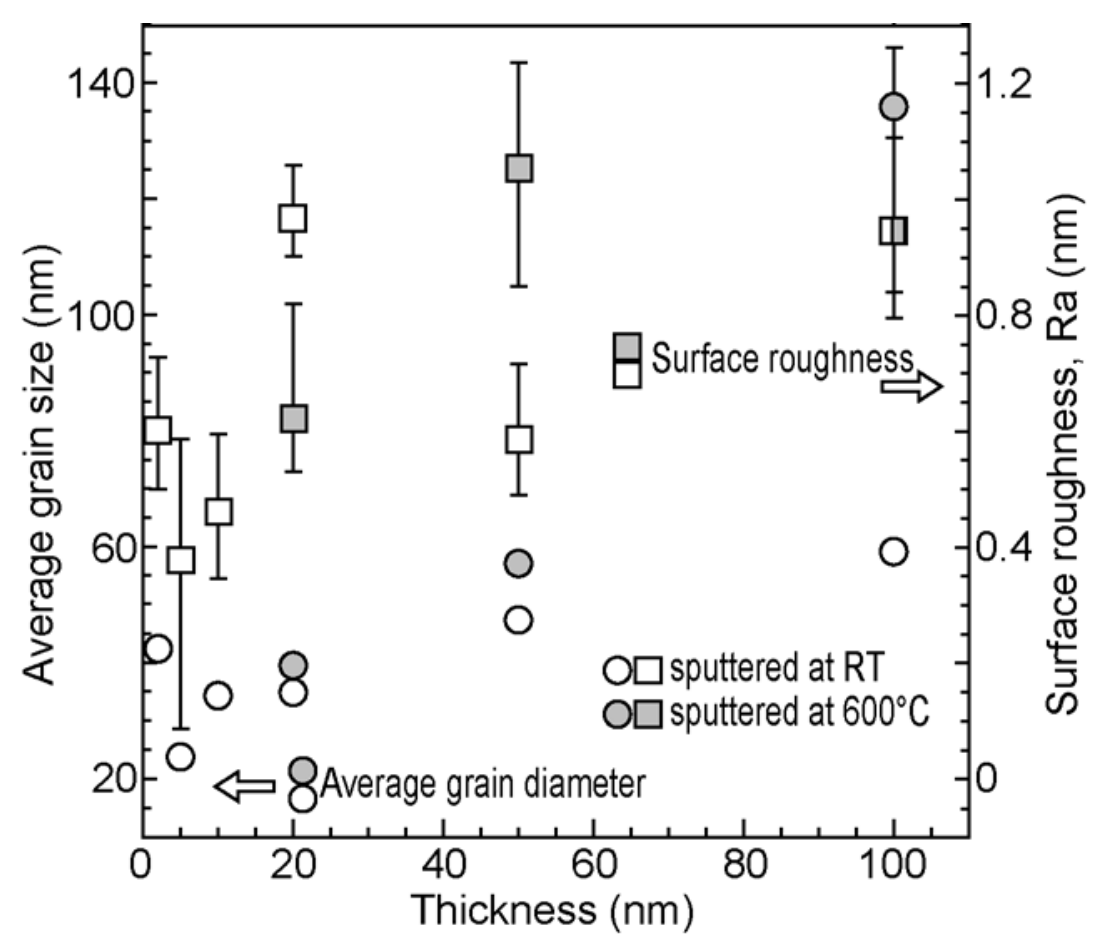

Fig. 2 

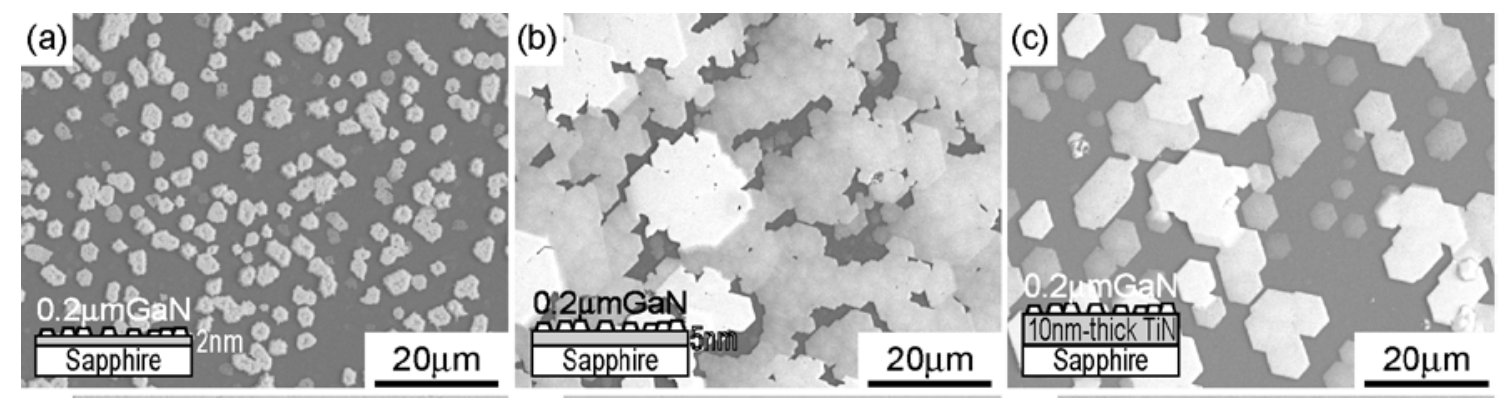

(d)

(e)

(f).

\begin{tabular}{|c|}
\hline 2umGaN \\
\hline \hline Sapphire \\
\hline
\end{tabular}

$20 \mu \mathrm{m}$

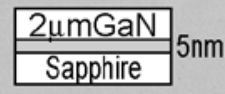

$20 u m$

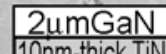

Fig. 3

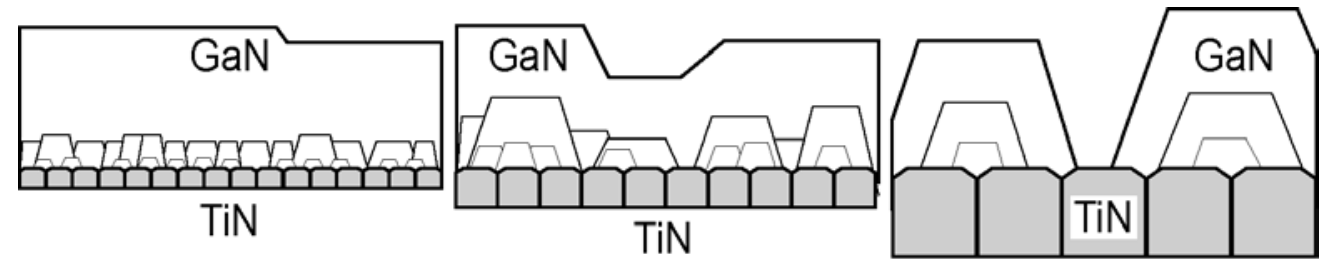

Fi.g4
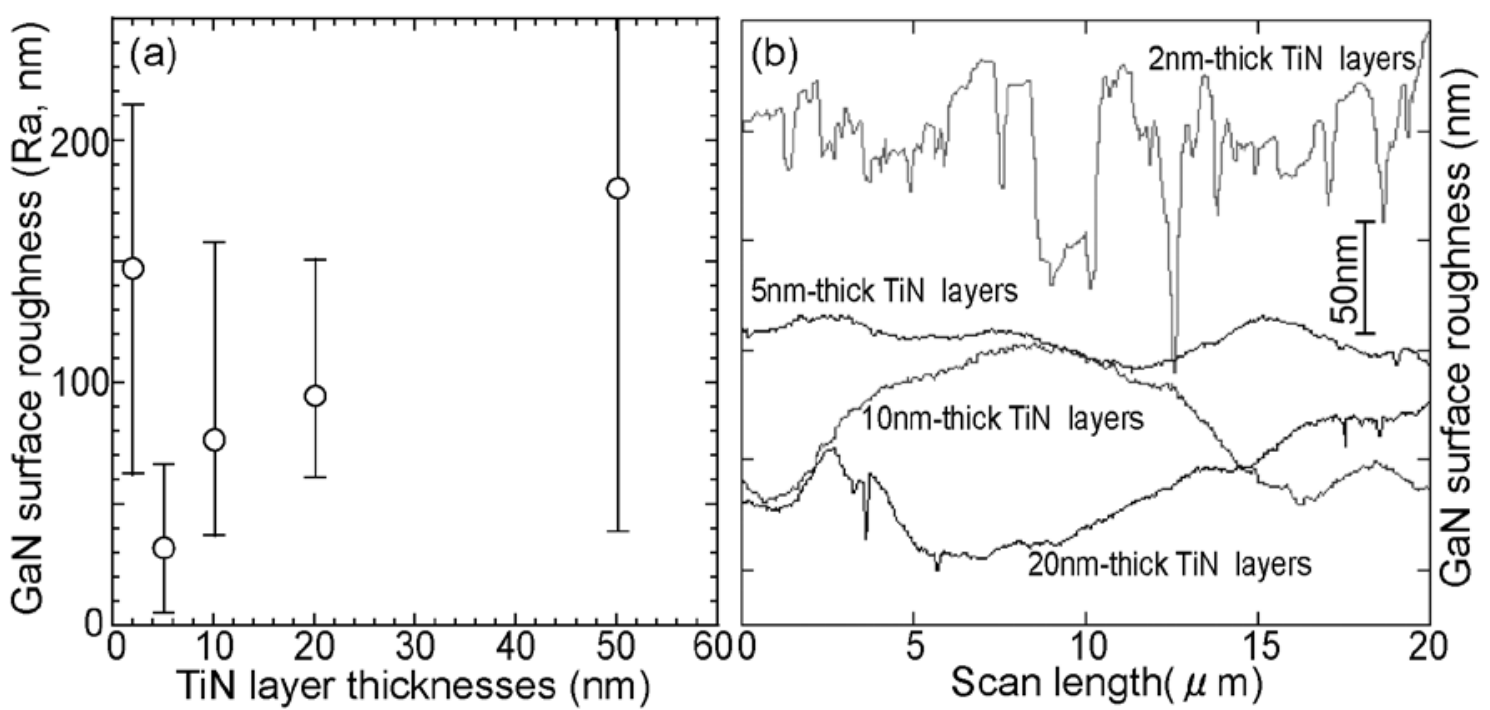

Fig.5 


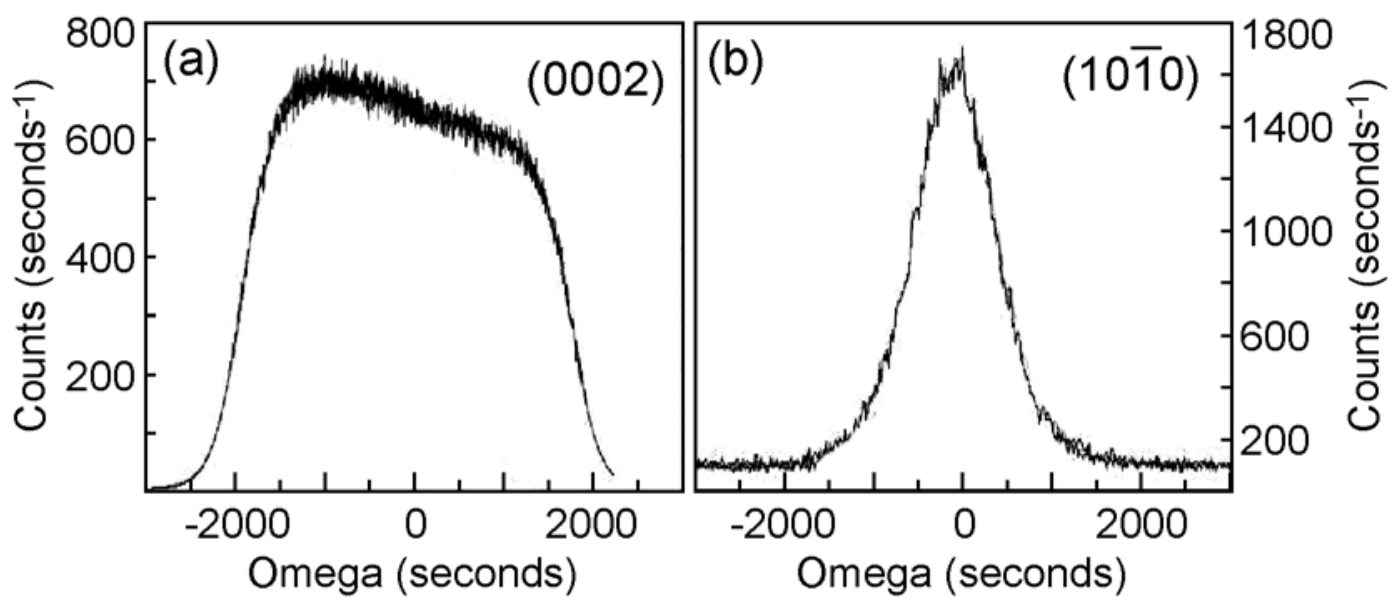

Fig. 6

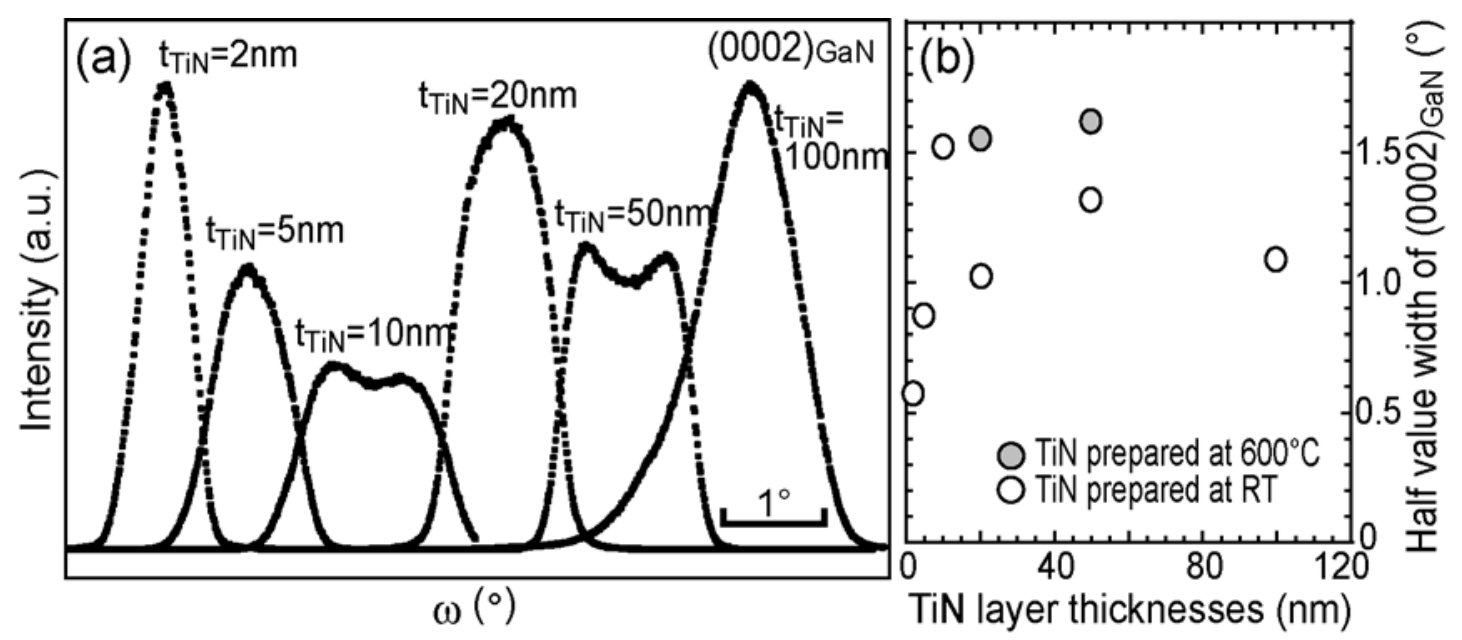

Fig.7 


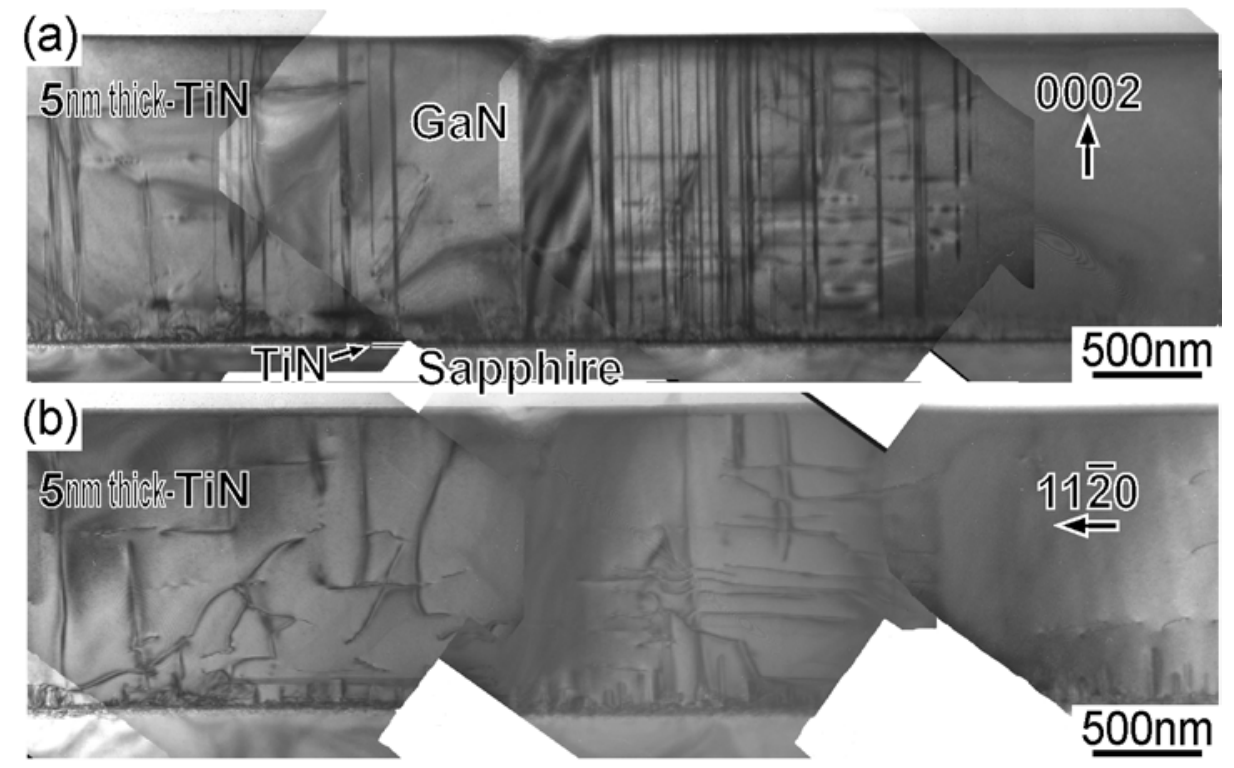

(c)

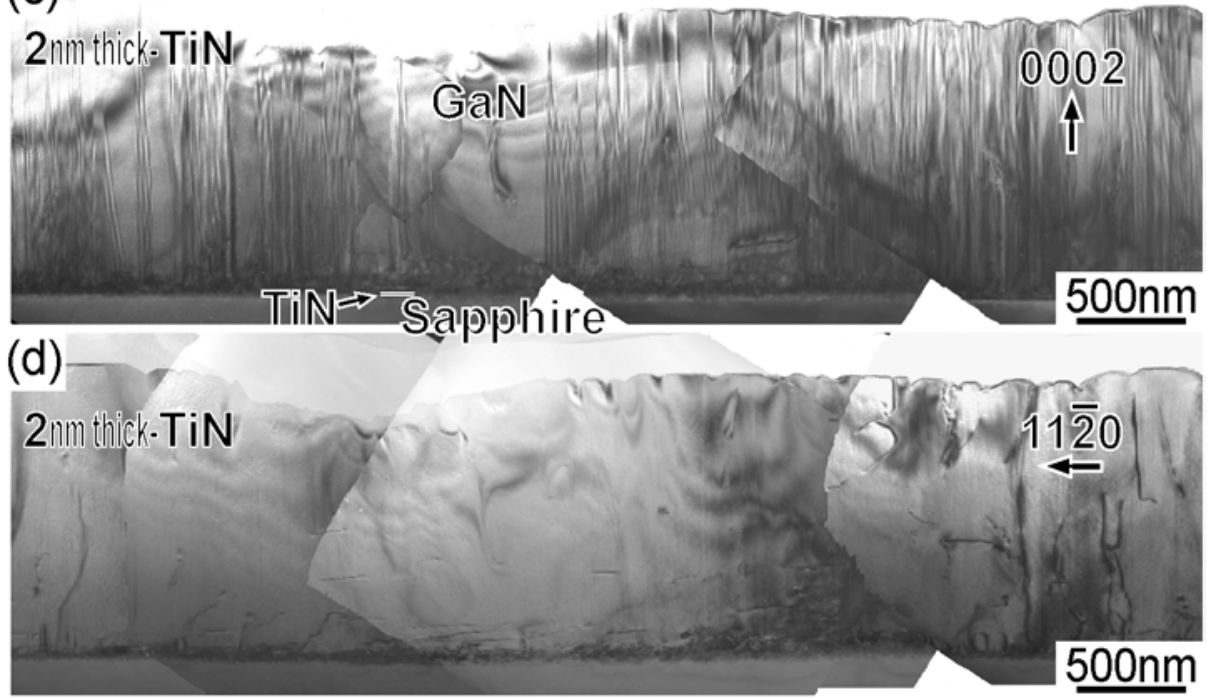

Fig. 8 